\title{
1918 - die Zeit der großen Schulreformprogramme in Österreich: Reformpädagogik und Geisteswissenschaftliche Pädagogik im Wettstreit
}

\section{Gerald GRIMM}

\section{ARTICLE INFO \\ Article history: \\ Received 20 February \\ 2019 \\ Accepted 24 May 2020 \\ Available online 31 July \\ 2020}

Keywords:

Austria 1918, school reform, New Education, Humanities Pedagogy, Otto Glöckel (18741935), Alpois Höfler (1853-1922), Richard Meister (1881-1964), Social Democrats, Christian Socials, one comprehensive school, equal opportunity, learning by doing, perpetuation of the diversity of types in secondary school level 1 , Haupt- and Mittelschulgesetz 1927

\footnotetext{
G. Grimm

University

of Klagenfurt

Department of School

Pedagogy and Historical

Educational Research •

Universitätsstraße 65-67

9020 Klagenfurt am

Wörthersee •

Austria •

Gerald.Grimm@aau.at
}

\begin{abstract}
1918 - the Time of Major School Reform Programs in Austria: New Education and Humanities Pedagogy in Competition

In 1918 Austria was changed into a democratic republic. Despite the difficult political and economic situation at the end of the First World War self-confident and future oriented school reform programs were developed in Austria. Influences of international New Education as well as of German Humanities Pedagogy can be determined. The Social Democrats under the leadership of Otto Glöckel, influenced by the ideas and concepts of New Education (such as "learning by doing"), postulated the creation of one comprehensive school ("Allgemeine Volksschule") up to the age of 14 in order to realize equal opportunity. The Christian Socials on the other side were influenced by ideas of the German Humanities Pedagogy and advocated under the Leadership of Alois Höfler and Richard Meister for maintaining the variety of types of secondary education at level I: "Volksmittelschule", "Untergymnasium", "Unterrealschule". Because of these great differences no agreement on a school reform program could be realized. Nevertheless in 1927 a compromise was decided: the "Haupt and Mittelschulgesetz". But type diversity in the field of secondary education remains and characterizes the Austrian school system even in the $21^{\text {st }}$ century.
\end{abstract}




\section{Die historisch-pädagogische Ausgangslage: Österreich 1918}

Die Niederlage Österreich-Ungarns im Ersten Weltkrieg war für die Bevölkerung der jungen Republik Österreich, des „Reststaates“ des zerfallenden alten Habsburgerreiches, zweifellos ein traumatisches Ereignis, das nie ganz überwunden wurde: „Die Erste Republik war geprägt davon, was sie von der Monarchie geerbt hatte, vielleicht mehr noch, was sie durch ihren Zerfall verloren hatte" (Höbelt, 2018, S. 11). Dennoch - oder gerade deshalb - wurden bereits 1918 Pläne entwickelt, die durchaus Selbstbewusstsein demonstrieren und auf eine positive Zukunft orientiert waren: „All diese Zukunftsentwürfe waren nämlich die längste Zeit vor allem einmal durch ihre Unerreichbarkeit gekennzeichnet. Man hielt an den Visionen fest, zweifellos; aber man musste sich inzwischen doch auch irgendwie über die Gegenwart hinweghelfen“ (2018, S. 12). Dies manifestiert sich insbesondere auf pädagogisch-bildungspolitischem Gebiet, wo die zeitgenössische internationale reformpädagogische Bewegung nun auch Österreich voll erfasste, wie dies die Beiträge österreichischer Gelehrter und Erziehungspraktiker zur Kunsterziehungsbewegung, zur Arbeitsschulbewegung und vor allem $\mathrm{zu}$ der sich primär in Wien konstituierenden Psychoanalytischen Pädagogik dokumentieren (vgl. Adam, 2007). Aber auch die in erster Linie im deutschen Sprachraum sich entfaltende Geisteswissenschaftliche Pädagogik wurde in Österreich rezipiert und fand vor allem an der Universität Wien prominente Fachvertreter, die sich gleich 1918 sogar bildungspolitisch betätigten.

Beide erziehungswissenschaftlichen Strömungen (Reformpädagogik und Geisteswissenschaftliche Pädagogik) entwarfen differente, aber großteils in die Zukunft weisende Schulreformprogramme für die junge Republik Österreich. Dabei intendierten die reformpädagogisch orientierten Kräfte um den Sozialdemokraten Otto Glöckel in Österreich wie die deutschen Reformpädagogen in der sich konstituierenden Weimarer Republik radikale Neuerungen, so etwa die „Demokratisierung der Schule, die Selbstverwaltung der Schüler und die Schaffung von Schulgemeinden als Lebensgemeinschaften“" (Seel und Hanke, 2015, S. 281); die Repräsentanten der sich an den deutschen Universitäten etablierenden Geisteswissenschaftlichen Pädagogik vertraten hingegen großteils eine eher konservative Position, ging es ihnen doch primär darum, „die alte Ordnung zu erhalten bzw. zu restaurieren“, da sie - trotz partieller Sympathien für reformpädagogisches Gedankengut wie etwa „Hermann [sic!] Nohl, Eduard Spranger und Erich Weniger der Weimarer Republik mit ausgesprochener Skepsis begegneten“ (2015, S. 281-282). Diese Affinität der Geisteswissenschaftlichen Pädagogik zu bürgerlich-konservativen Positionen ist auch in Österreich zu konstatieren; dennoch plädierten deren Vertreter in Österreich, insbesondere Alois Höfler und Richard Meister, für eine evolutionäre Weiterentwicklung des Bildungswesens. Aus diesen Gründen gab es in Detailfragen durchaus auch Übereinstimmungen zwischen beiden erziehungswissenschaftlichen Strömungen (vgl. Reble, 1989, S. 357 mit Bezug auf Meister; konkret etwa bei der Reform des Volksschullehrplanes 1926; vgl. Grimm, 2008, S. 300). Damit ist auch das Zustandekommen des Schulkompromisses in Österreich im Jahre 1927 zu erklären. Diametral entgegengesetzt 
waren freilich die Positionen hinsichtlich der Neugestaltung der Sekundarstufe I, wo es bereits $1918 \mathrm{zu}$ einem regelrechten Wettstreit zwischen beiden pädagogisch-politischen Richtungen kam.

\section{Reformpädagogik und Sozialdemokratie: das Schulreformprogramm Otto Glöckels}

Die intensive Schulreformdiskussion begann in Österreich schon während des Ersten Weltkrieges. Am 7. November 1917, als sich die Niederlage der Mittelmächte bereits abzuzeichnen begann, hielt Otto Glöckel (1874-1935), sozialdemokratischer Reichsratsabgeordneter, im Großen Konzerthaussaal in Wien eine programmatische bildungspolitische Rede mit dem Titel das „Tor der Zukunft“. Der 1874 als Sohn eines Volksschullehrers im niederösterreichischen Pottendorf geborene Glöckel hatte nach seiner Ausbildung zum Pflichtschullehrer am Landeslehrerseminar in Wiener Neustadt fünf Jahre, und zwar von 1892 bis 1897, als „Unterlehrer“ an einer Volksschule in Wien unterrichtet. Er trat dem liberal orientierten, überparteilichen und bereits den Prinzipien der zeitgenössischen internationalen Reformpädagogik verpflichteten Verein „Freie Schule“ bei; darüber hinaus engagierte Glöckel sich in der Sozialdemokratischen Partei. Die Reichshauptstadt Wien wurde jedoch seit Mitte der 1890er Jahre von der Christlichsozialen Partei regiert. Als Bürgermeister fungierte seit 1897 Karl Lueger, der kurz nach seinem Amtsantritt, und zwar am 15. September 1897, „ohne jedes Verfahren und ohne Angabe von Gründen, fünf provisorische Unterlehrer, darunter auch Otto Glöckel“ (Achs und Krassnigg, 1974, S. 49-50.) fristlos kündigte. Glöckel fand zwar ziemlich rasch ein neues berufliches Betätigungsfeld (Beamter bei der Arbeiter-Krankenkasse sowie der Arbeiter-Unfallversicherungsanstalt), dennoch blieben die Schul- und Bildungspolitik sowie die damit verbundenen sozial- und gesellschaftspolitischen Fragen weiterhin sein Hauptinteressensgebiet. Seit 1907 war er deshalb als Reichsratsabgeordneter der Sozialdemokratischen Arbeiterpartei und gleichsam als deren „Bildungssprecher“ tätig. Glöckel vereinigte außerdem den reformpädagogisch orientierten Verein „Freie Schule“ mit dem 1908 von Anton Afritsch als Vorfeldorganisation der Sozialdemokratischen Partei gegründeten Vereinigung „Die Kinderfreunde“, der ebenfalls für eine demokratische und sozial gerechte, von der Kirche unabhängige (Pflicht-)Schule eintrat (vgl. Gönner, 1967, S. 187). Damit begann die für Österreich resp. Wien so charakteristische Symbiose von Reformpädagogik und Sozialdemokratie. Diese manifestierte sich vor allem in der Debatte um die „Einheitsschule“, der gemeinsamen Schule der Sechs- bis Vierzehnjährigen, also der einheitlichen Organisation von Primarstufe sowie Sekundarstufe I. Glöckel thematisierte diese brisante und dominante bildungspolitische Streitfrage der Ersten Republik Österreich bereits im September 1916 in einem Artikel in der „Arbeiter=Zeitung“, dem „Zentralorgan der Deutschen Sozialdemokratie in Oesterreich", wobei er parteipolitische Postulate (Chancengerechtigkeit) und reformpädagogische Prinzipien (Lebensnähe) miteinander kombinierte: „Bis zum fünfzehnten Lebensjahr reicht für alle Fälle eine Schulart völlig aus. Wir brauchen die Einheitsschule! Im fünfzehnten Jahre soll erst entschieden werden, ob der junge Mensch die Fähigkeit besitzt, um weiterstudieren zu können, oder ob er neben der Erlernung eines Praktischen Berufes die obligate Fortbildungsschule besuchen soll. Die 
Entscheidung darf allerdings nicht danach erfolgen, welche soziale Stellung die Eltern einnehmen [...] Wir brauchen also eine Einheitsschule ohne Berechtigungswesen; ein vernünftiger Lehrplan ist notwendig, der auf die Bedürfnisse des Lebens Rücksicht nimmt“ (Glöckel, 1916/1985, S. 92-94). Glöckel avanciert damit zu einem der Wegbereiter der Gesamtschulidee in Österreich (vgl. Severinski, 1985, S. 109-141).

Auch in der bereits erwähnten Rede „Das Tor der Zukunft“, die übrigens Ende 1917 im Verlag des Vereines „Freie Schule“ in Broschürenform publiziert wurde, ist dieses Nebeneinander parteipolitisch motivierter Argumente und reformpädagogischer Prinzipien zu konstatieren. Sie stellt zweifellos den Höhepunkt von Glöckels bildungspolitischem Wirken in der Habsburgermonarchie dar. Der Fokus liegt darin bei der Orientierung aller Schulreformvorhaben am Wohle des Kindes. Glöckel erwähnt in diesem Zusammenhang explizit Ellen Keys programmatische Schrift „Das Jahrhundert des Kindes“ (Glöckel, 1917/1985, S. 104) und hebt die Bedeutsamkeit der Schule als „Tor einer glücklichen Zukunft“ für das Individuum, aber auch für Staat und Gesellschaft, hervor: „Eine neue Zeit soll vorbereitet werden, gerechter, fruchtbarer, schöner!“ (1917/1985, S. 102). Aus diesen Erwägungen postuliert der sozialdemokratische Reichsratsabgeordnete eine grundlegende Reorganisation des gesamten österreichischen Bildungswesens von den Kinderkrippen, Kinderheimen und Kindergärten bis zu den Universitäten. Er zitiert in diesem Zusammenhang den bayerischen Schulreformer und Reformpädagogen Georg Kerschensteiner (1917/1985, S. 108), der schon im ersten Dezennium des 20. Jahrhunderts den Aufbau eines gänzlich neuen Schulwesens gefordert hatte und ein Wegbereiter der Arbeitsschulbewegung, einer der einflussreichsten Teilströmung der Reformpädagogik, war. Im Mittelpunkt der geplanten Reformvorhaben steht für Glöckel freilich die Überwindung der ,verwirrenden Mannigfaltigkeit unseres Schulwesens“ durch Schaffung einer „Einheitsschule“ bzw. „Allgemeinen Volksschule“ bis zur Vollendung der Schulpflicht im 15. Lebensjahr. Unter Bezugnahme auf den deutschen (Sozial-)Pädagogen Paul Natorp soll diese Einheitsschule jedoch nicht einer „mechanischen Gleichmachung“ aller Kinder und Jugendlichen dienen, sondern vielmehr durch innere Differenzierung den Begabungsschwerunkten der Schülerinnen und Schüler Rechnung tragen (1917/1985, S. 109). Parallel dazu fordert der engagierte Sozialdemokrat Glöckel sozialpolitische Maßnahmen wie die Abschaffung des Schulgeldes, also die „volle Unentgeltlichkeit des Unterrichts“, weiters „Lehrmittelfreiheit“ sowie den Ausbau des Stipendienwesens (1917/1985, S. 110-112.), um Chancengerechtigkeit im Bildungswesen wenigsten ansatzweise zu realisieren.

Methodisch-didaktische Reformen waren Glöckel ein besonderes Anliegen. Hier erweist er sich wiederum als Sympathisant der Reformpädagogik, wenn er in diesem Zusammenhang die freie Methodenwahl durch den Lehrer sowie die Verbannung jeglichen Drills aus dem Schulunterricht fordert. Denn nur so könne die Schule die so essentielle Lernfreudigkeit erzeugen und selbständiges Lernen im Sinne der Arbeitsschule Kerschensteiners fördern. Wie der deutsche Reformpädagoge Peter Petersen später (in den 1920er Jahren) in seinem 
Jenaplan betont auch Glöckel den Wohnstubencharakter des Klassenraums und fordert die Lehrer/-innen auf, ,aus der Schulstube einen festlichen Raum zu machen, ein wenig Glanz, ein wenig wärmenden Sonnenschein in die sonst trüben Tage der Jugend zu bringen“ (1917/1985, S. 114). Eine grundlegende Reform der Lehrer/-innenbildung ist für den sozialdemokratischen Bildungspolitiker und Reformpädagogen Glöckel überhaupt die Conditio sine qua non einer erfogreichen Schulerneuerung. Dabei erscheint ihm die hochschulmäßige Ausbildung auch der künftigen Pflichtschullehrer/-innen unbedingt erforderlich $\mathrm{zu}$ sein, um die intendierte Qualitätsverbesserung des Elementarunterrichts realisieren zu können: „wir verlangen, daß der Lehrer eine Obermittelschule besucht habe, daß ihm das Hochschulstudium ermöglicht werde. Nicht weltfremde ,Schulgehilfen“ wollen wir, sondern aufrechte, kluge Männer und Frauen sollen unsere Kinder fürs Leben vorbereiten“ (1917/1985, S. 117). Die materielle Unabhängigkeit des Lehrerstandes durch adäquate Entlohnung und ein Dienstrecht, das den Lehrer/-innen, die freie, unparteiische Berufsausübung ermöglicht, sind weitere Postulate Glöckels. Außerdem fordert er die vollständige „Trennung von Kirche und Staat“ auch auf dem Gebiet des Bildungswesens, ,der Religionsunterricht soll aber ausschließlich eine Angelegenheit der Kirche bleiben, dem Staat obliegt nur das Aufsichtsrecht in methodischer und sachlicher Beziehung“ (1917/1985, S. 105). Schließlich plädiert der Sozialdemokrat und Reformpädagoge Glöckel für das Mitspracherecht der Eltern in Schulangelegenheiten, wofür auch der Verein „Freie Schule“ als eine „Elternvereinigung“ eintrete (1917/1985, S. 117). Damit waren Ende 1917 die Grundlinien einer von reformpädagogischen Prinzipien geleiteten österreichischen Schulreform unter sozialdemokratischer Federführung, die sich darüber hinaus den Leitideen Demokratisierung und Liberalisierung verpflichtet fühlte (Grimm, 2007, S. 48-50), gezeichnet worden. Das bürgerlich-konservative Lager und die akademische Pädagogik waren damit jedenfalls herausgefordert und mussten reagieren.

\section{Die Geisteswissenschaftliche Pädagogik als Alternative: Alois Höflers Vorschläge zur Neugestaltung des österreichischen Schulwesens}

Die österreichischen resp. Wiener Hochschullehrer meldeten sich noch im Jahre 1917 mit diversen Initiativen zu Wort. Diese legten ihren Fokus auf eine „Verbesserung des Mittelschulunterrichts“, also die methodisch-didaktische und curriculare Neugestaltung der zur Hochschulreife führenden Sekundarschulen (Gymnasien, Realschulen und Realgymnasien; vgl. Höfler, 1918, S. 4). Ihr Wortführer war der Ordinarius für „Pädagogik einschließlich Philosophie“ an der Universität Wien, Alois Höfler (1853-1922). Der 1853 als Sohn eines Juristen im oberösterreichischen Kirchdorf an der Krems geborene Höfler hatte an der Universität Wien ein Lehramtsstudium in Mathematik und Physik absolviert sowie zusätzlich die Lehramtsprüfung für das Fach Philosophische Propädeutik abgelegt. Von 1877 bis 1903 war er als Gymnasiallehrer für diese Fächer in Wien tätig und ist schon in dieser Zeit als Verfasser von Beiträgen zur Mittelschulreform publizistisch hervorgetreten. 1895 habilitierte sich Höfler an der Universität Wien für Philosophie und Pädagogik und nahm im Sommersemester dieses Jahres seine akademische Lehrtätigkeit auf. 1903 wurde Höfler als 
Nachfolger Otto Willmanns als Professor für Pädagogik an die Deutsche Universität Prag berufen, obwohl er, wie Wolfgang Brezinka kritisch resümiert, „kein Pädagogiker, sondern der damals bedeutendste Didaktiker und Reformator des Unterrichts in Physik und Philosophischer Propädeutik war“ (Brezinka, 2003, S. 89). Vier Jahre später (1907) wechselte Höfler als Nachfolger Theodor Vogts an die Lehrkanzel für Pädagogik an der Universität Wien, die er bis zu seinem Tode im Jahre 1922 innehatte. Mit der Berufung Höflers nach Wien begann zweifellos ein neues Kapitel in der Geschichte der akademischen Pädagogik in Österreich. Denn bis 1907 dominierte der Herbartianismus in der Habsburgermonarchie. Höfler verband jedoch Pädagogik mit neueren philosophischen Ansätzen (namentlich seien hier Franz Brentano und Alexius Meinong genannt, vgl. Höfler, 1921, S. 5-7.) und betrieb darüber hinaus „,ächerübergreifende Forschung und Lehre inkl. experimenteller Psychologie“ (Dahms und Stadler, 2015, S. 82). Damit avancierte Höfler zum Wegbereiter einer neuen, stärker geisteswissenschaftlich akzentuierten Pädagogik (Verbindung von Pädagogik mit Philosophie, Logik und Ethik und experimenteller Psychologie; vgl. Brezinka, 2000, S. 318) in Österreich, deren Blütezeit im deutschen Sprachraum dann in die Zwischenkriegszeit fallen sollte.

Seit seiner Berufung zum Universitätsprofessor widmete sich Höfler immer mehr auch aktuellen Fragen der Schulreform in Österreich. Der Schwerpunkt seiner einschlägigen Beiträge lag hier bei der Reform der Gymnasien. Der Pädagogikprofessor plädierte bereits 1906 für die stundenmäßige Ausweitung des Unterrichts an den österreichischen Gymnasien und postulierte die Aufnahme einer modernen Fremdsprache (Französisch oder Englisch) als Pflichtgegenstand in den Lehrplan des Gymnasiums - beides auf Kosten des Griechischen (vgl. Höfler, 1906, S. 635-636.). Damit avancierte Höfler zum geistigen Wegbereiter des Realgymnasiums als Langform, welches im Zuge der Mittelschulreform von 1908 auch in Österreich Realität wurde. Auch in den Jahren des Ersten Weltkrieges meldete sich der Wiener Pädagogikprofessor mit Beiträgen zur Zukunft des höheren Schulwesens in Österreich zu Wort (vgl. Höfler, 1916). Den Höhepunkt von Höflers bildungspolitischem Engagement stellt aber zweifellos die 1918 publizierte Schrift „Das Ganze der Schulreform in Österreich“ dar. Schon in der Titelwahl ist die Kritik an dem auf die Neugestaltung der Sekundarstufe I fokussierten, reformpädagogisch akzentuierten Schulreformprogramm der Sozialdemokraten erkennbar. Höfler plädiert darin nämlich für eine ,großzügige Reform“, die ihren Blick auf „das Ganze“ des Bildungswesens richtet (Höfler, 1918, S. 61). Um dem „Schulelend“ der Gegenwart, dem „multum necessarium unserer Volks-, Bürger-, Mittel- und Hochschulen“ (1918, S. 66) ein Ende zu bereiten, fordert der Wiener Pädagogikprofessor Reformen in allen Bereichen und Sektoren des österreichischen Bildungswesens. Höfler plädiert insbesondere für die „Weiterentwicklung unserer verunglückten ,Bürgerschule“ [Volksschuloberstufe im städtischen Raum, GG] zu einer wahrhaften ,Volksmittelschule““ (1918, S. 66). Die Typenvielfalt im Bereich der Sekundarstufe I möchte Höfler freilich beibehalten und postuliert vielmehr die Verabschiedung eines einheitlichen „Reichsmittelschulgesetzes“, das nach Kriegsende das österreichische Mittelschulwesen (Gymnasien, Realschulen und 
Realgymnasien) auf völlig neue legistische Grundlage stellen sollte (1918, S. 69-90). Dieses sollte nach Ansicht des Wiener Professors unbedingt auch die grundlegende Neugestaltung der Mittelschullehrerbildung inkludieren. Die Hauptform im Bereich der Sekundarstufe I sollte künftig die bereits erwähnte neue „,vierklassige Volksmittelschule“ sein, die sich unmittelbar an den Primarbereich, die vierklassige Volksschule anschließt. Die Untergymnasien und Unterrealschulen bleiben allerdings bestehen und bereiten unmittelbar auf die Oberstufenformen der höheren Schulen vor. Die Absolventen der Volksmittelschulen können in „Fachschulen aller Art“ oder „unmittelbar in das Erwerbsleben“ wechseln, müssen in diesem Fall aber parallel die obligaten Fortbildungsschulen besuchen (1918, S. 70-71). Eine „Einheitsschule“ der Sechs- bis Vierzehnjährigen, wie Glöckel sie vorschwebte, lehnt Höfler somit entschieden ab. Der Wiener Pädagogikprofessor führt für sein entschiedenes Veto gegen die Einheitsschule primär sozio-ökonomische Argumente an: „Die weitestgehende Auslegung dieses vieldeutigen Wortes [Einheitsschule, GG] würde voraussetzen, daß von sämtlichen Angehörigen eines Staates im Berufsleben wesentlich gleichartige oder geleichwertige Leistungen erwartet werden und daß daher auch die Vorbildung wie in der Volks- so auch in der Mittelschule (d. h. höchstens in der Untermittelschule, jedenfalls nicht mehr in der Hochschule) dieselbe sein müsse; eine „,solche Annahme von Gleichheit der Leistungen aller Staatsbürger" ist aber nach Ansicht Höflers „durch die tatsächliche Mannigfaltigkeit der Staatsbedürfnisse als völlig lebensfremd von vornherein widerlegt“" (1918, S. 71). Ohne das Glöckelsche Reformprogramm explizit zu erwähnen, kritisiert Höfler die von Sozialdeorraten postulierte Vereinheitlichung der Sekundarstufe I scharf und sieht vielmehr die Realisierung der ,auch künftig nicht herabzusetzenden Bildungsziele der Obermittelschule“, womit er insbesondere die Hochschulreife meint, „durch ein unorganisches Aufsetzen der Obermittelschulen auf eine undifferenzierte Einheits-Untermittelschule“ (1918, S. 72) akut gefährdet.

Das von Höfler formulierte Reformprogramm basiert auf einem Pädagogikverständnis, dem zweifellos das Attribut ,geisteswissenschaftlich“ zuerkannt werden kann. Für ihn gibt es nämlich keine wissenschaftliche Pädagogik, „die nicht ganz und gar wurzelte in Psychologie, Logik und Ethik, also Philosophie“ (1918, S. 171). Dabei nimmt die Psychologie in Gestalt der „Dispositionspsychologie“ die führende Rolle ein, denn „Erziehung ist die planmäßige Ausbildung wertvoller Dispositionen des Zöglings“, weshalb für Höfler sich „,der größte Teil der Pädagogik sogar mit Dispositions-Psychologie deckt“ (Höfler, 1908, S. 131). Sein Reformprogramm von 1918 enthält auch durchaus innovative Vorschläge. In diesem Zusammenhang sei insbesondere das erste Modell einer Zentralmatura in der österreichischen Schulgeschichte erwähnt (vgl. Höfler, 1918, S. 151-169; dazu Grimm, 2018, S. 155-160). Aber auch Höflers Gedanken zur Reform der Lehrerbildung waren 1918 durchaus in die Zukunft weisend: bessere pädagogische Vorbildung der künftigen Mittelschullehrer an pädagogischen Universitätsinstituten sowie Reform der Pflichtschullehrer/-innenbildung durch Weiterentwicklung der Lehrer- und Lehrerinnenbildungsanstalten zu vierjährigen „Lehrermittelschulen“ (Sekundarstufe II), 
an die noch zweijährige „Lehrerakademien“ anschließen sollten (vgl. Höfler, 1918, S. 169179). In bürgerlich-konservativen Kreisen fanden Höflers Schulreformvorschläge jedenfalls starke Resonanz, weshalb es nicht verwunderlich erscheint, dass die Christlichsoziale Partei, die im November 1918 eine „Zerreißprobe“ (drohende Spaltung in einen monarchischen und republikanischen Flügel; vgl. Kriechbaumer, 2017, S. 137) zu bestehen hatte, diese weitgehend übernahm. Massive bildungspolitische Konflikte zwischen den beiden großen ideologischen Lagern (Sozialdemokraten auf der einen, Christlichsoziale auf der anderen Seite) waren deshalb am Beginn der Ersten Republik Österreich gleichsam vorprogrammiert.

\section{Zur Schulreformdiskussion in der Ersten Republik Österreich mit einem Ausblick auf die Gegenwart}

Am 16. Februar 1919, drei Monate nach Ausrufung der Ersten Republik Österreich am 12. November 1918, wurden die ersten Wahlen nach Kriegsende durchgeführt. Die Sozialdemokraten gingen dabei als knappe Sieger hervor. Die Bildung einer großen Koalition mit der zweitstärksten politischen Kraft, den Christlichsozialen, war die Folge. Otto Glöckel wurde als „Unterstaatssekretär“ im neu gegründeten „Staatsamt für Inneres und Unterricht“ mit der Leitung der Schulagenden betraut und damit de facto Unterrichtsminister. Eine seiner ersten Maßnahmen war ein mit 10. April 1919 datierter Erlass der jeden Zwang zur Teilnahme von Schülerinnen und Schülern an religiösen Übungen untersagte und auch bestimmte, dass ,die Nichtteilnahme auf die Klassifikation des Schülers keinen Einfluß haben dürfe“ (Dachs, 1982, S. 40). Diese als „Glöckel-Erlass“ in die Schulgeschichte der Ersten Republik eingegangene Verfügung hatte in der ,hohen Zeit der bildungspolitischen Ideologien“ (vgl. Lechner, 1995) weit reichende Konsequenzen. Einerseits belastete der Glöckel-Erlass das ohnehin konfliktgeladene Verhältnis von Sozialdemokratie und katholischer Kirche und hatte andererseits negative Konsequenzen für das Koalitionsklima, auch wenn die christlichsozialen Minister nicht offen gegen diese Anordnung des Unterstaatssekretärs für Unterricht protestierten.

Am 22. April 1919 präsentierte de-facto-Unterrichtsminister Glöckel den Ausschuss für Erziehung und Unterricht sein Schulreformprogramm. Dieses basierte in der Hauptsache auf seiner bereits zitierten Rede „das Tor der Zukunft“ vom Herbst 1917. Als „Grundsätze für die Schulreform“ nannte Glöckel unter expliziter Berufung auf die internationale Reformpädagogik „das Recht jedes Kindes auf Ausbildung und Auswertung all seiner Fähigkeiten und Talente“ sowie die Prinzipien der „Einheitsschule“ bis zum 14. Lebensjahr und der „Arbeitsschule“ (Glöckel, 1919/1985, S. 141-143). Mit „Arbeitsschule“ meinte Glöckel nicht einen neuen Schultyp, sondern in Anlehnung an den bayerischen Reformpädagogen Georg Kerschensteiner ein methodisch-didaktisches Reformmodell (vgl. Hackl, 1990), in dessen Mittelpunkt die Aktivierung der Lernenden im Unterricht, also die weitgehend selbständige Erarbeitung des Lehrstoffes durch die Schülerinnen und Schüler selbst, steht: „Die Arbeitsschule sucht das Problem zu lösen, alle Sinne des jungen Menschen in den Dienst des Unterrichtes zu stellen, die Erkenntnisse soweit als möglich selbst 
zu erarbeiten. Das Problem der Arbeitsschule ist also zunächst eine Frage der Lehrmethode“ (Glöckel, 1919/1985, S. 143). Das von Glöckel formulierte sozialdemokratische Schulreformkonzept kann jedenfalls als eine „Synthese verschiedener Schulorganisationspläne und reformpädagogischer Überlegungen unterschiedlicher, vor allem deutscher Herkunft, jedoch auf die spezifischen Verhältnisse in Österreich bezogen“ (Scheipl, 2017, S. 323) bezeichnet werden. Bemerkenswert erscheint in diesem Zusammenhang die Forderung Glöckels, dass ,bei allen Fragen der Schulreform [...] die größte Angleichsmöglichkeit an Deutschland ins Auge gefaßt werden muß“ (Glöckel, 1919/1985, S. 146). Mit diesem Postulat ging übrigens der Unterstaatssekretär für Unterricht mit den allgemeinen politischen Zielvorstellungen seiner Partei konform, blieb doch der Anschluss der Republik an das Deutsche Reich „,bis 1933 im Parteiprogramm der Sozialdemokratischen Arbeiterpartei Österreichs verankert“ (Engelbrecht, 1988, S. 10).

Mit der Christlichsozialen Partei konnten Glöckel und die Sozialdemokraten auf Grund ihres liberalen, reformpädagogisch akzentuierten Schulreformprogramm keinen Konsens erzielen, so dass im Bereich des Bildungswesens die gesetzlichen Bestimmungen aus der Zeit der Habsburgermonarchie in den ersten Jahren der Ersten Republik Österreich bestehen blieben. Im Sommer 1920 beendeten dann die Christlichsozialen die Zusammenarbeit mit den Sozialdemokraten. Bei den Neuwahlen am 17. Oktober 1920 wurden die Christlichsozialen stimmenstärkste Partei und die Sozialdemokraten gingen in die Opposition. Während Glöckel in den 1920er Jahren versuchte, sein Schulreformkonzept zumindest in der sozialdemokratisch regierten Bundeshauptstadt Wien, die $1921 \mathrm{zu}$ einem selbständigen österreichischen Bundesland aufstieg, umzusetzen (vgl. Grimm, 2007, S. 58-64), wurde auf Bundesebene die Bildungspolitik nunmehr weitgehend von den Christlichsozialen bestimmt. Deshalb meldeten sich auch wiederum die an der Universität Wien tätigen Repräsentanten der Geisteswissenschaftlichen Pädagogik in Österreich zu Wort. Gemeinsam mit Richard Meister (1881-1964), seit 1920 Professor für Klassische Philologie an der Universität Wien und ab 1923 sein Nachfolger als Ordinarius für Pädagogik, legte Alois Höfler 1921 den „Entwurf eines vollständigen Volks- und Mittelschulgesetzes“ vor. Dieser wurde „vom Akademischen Senat der Wiener Universität unterstützt“ (Wallraf, 1986, S. 158) und unter dem bezeichnenden Titel „Audiatur et altera pars“ als Alternative zu den sozialdemokratischen Schulreformbestrebungen veröffentlicht. Höfler und Meister plädierten darin für die Beibehaltung eines differenzierten Schulsystems im Bereich der Sekundarstufe I, wobei neben der Volksschuloberstufe vier Formen der Mittelschule, und zwar Volksmittelschule, Realschule, Lateinrealschule resp. Realgymnasium sowie Gymnasium, vorgesehen waren, zu denen noch die „Lehrermittelschule“ und die „Mädchenobermittelschule“ in der Sekundarstufe II traten (vgl. Höfler und Meister, 1921, S. 283-292, 311-323, 479-493).

Nach Höflers Tod im Jahre 1922 wurde Meister dann der „Hauptträger der wissenschaftlichen Auseinandersetzungen mit den sozialdemokratischen Schulreformversuchen" (Engelbrecht, 1988, S. 93). Meister, der bis 1956 an der Universität Wien lehrte, avancierte jedenfalls zum 
Hauptvertreter der Geisteswissenschaftlichen Pädagogik in Österreich, die er zu einer partiell durchaus innovativen „Kulturpädagogik“ erweiterte (vgl. Ballauff und Schaller, 1973, S. 682-684; Wallraf, 1986, S. 91-135; Brezinka, 2000, S. 443-453. Sie stellt wohl keinen „Weg in die Erstarrung durch Konservatismus“ (so Olechowski, 2014) darl. Die von Meister konzipierte kulturphilosophische Pädagogik ,war um eine systematische Vereinigung der traditionellen Kulturpädagogik und der Kulturphilosophie bemüht“" (Engelbrecht, 1988, S. 54), wobei ein signifikanter Einfluss Eduard Sprangers zu konstatieren ist, den der Wiener Professor auch zitiert (vgl. Meister, 1924/1946, S. 14). In methodisch-didaktischer Hinsicht spielte dabei wie bei Höfler die Psychologie eine essentielle Rolle, werden doch Unterrichtsfächer von Meister unter Bezugnahme auf seinen Amtsvorgänger als „Dispositionssysteme“ (vgl. Meister 1919/1946, S. 76-91) resp. als „Konzentrationsfelder didaktischer Arbeit“ betrachtet (vgl. Meister 1921/1946, S. 69-75). Bemerkenswert erscheint, dass „diese Formulierungen auch in die Lehrpläne der österreichischen Mittelchulen von 1928 und 1933 eingegangen sind, deren ,Allgemeiner Teil‘, eine Pädagogik, in nuce', ja zum größten Teil von Meister selbst verfaßt worden ist" (Hörburger, 1967, S. 190). Dies dokumentiert den großen Einfluss, den die Geisteswissenschaftliche Pädagogik auf die österreichischen Schulreformen in der Ersten Republik ausgeübt hat. Richard Meister zählt überhaupt ,zu den wenigen Erziehungswissenschaftlern, deren theoretische Überlegungen nicht nur in akademischen Vorlesungen ihren Niederschlag fanden, sondern auch Gesetze und Verordnungen der Schulverwaltung zu beeinflussen imstande waren“ (Engelbrecht, 1988, S. 52).

Da das österreichische Bundesverfassungsgesetz von 1920 für Schulgesetze eine Zwei-Drittel-Mehrheit vorsah, war die Zustimmung beider großer Parteien - der Sozialdemokraten und der Christlichsozialen - erforderlich (vgl. Grimm, 2008, S. 295-297). Ein solcher Kompromiss schien zunächst in weiter Ferne zu liegen; vielmehr wurde in Österreich in den 1920er Jahren ein „leidenschaftlicher Schulkampf“ (Scheipl, 2017, S. 323) geführt. Obwohl Reformpädagogik und Geisteswissenschaftliche Pädagogik und die sie jeweils unterstützenden politischen Großparteien bisweilen durchaus konstruktiv miteinander kommunizierten, ging es ,in der Dialogpraxis nicht immer glimpflich ab, insbesondere dann nicht, wenn fundamentale Glaubensüberzeugungen eines Dialogpartners [hier Glöckels Konzept einer Einheitsschule der Sechs- bis Vierzehnjährigen] ins kritische Visier des anderen“ gerieten (Skiera, 2018, S. 104). Am 2. August 1927 wurde im Nationalrat dennoch „weitgehend einstimmig“ (Engelbrecht, 2014, S. 49) das Haupt- und Mittelschulgesetz beschlossen. Wie bei einem Kompromiss üblich, hatten beide Seiten „Opfer“ gebracht: Die Sozialdemokraten verzichteten auf die „Allgemeine Mittelschule“ als einheitliche Schulform der Zehn- bis Vierzehnjährigen, sahen aber in der neuen, in zwei Klassenzügen geführten Hauptschule, welche die aus der Monarchie tradierte Bürgerschule ablöste, die Weichen in Richtung Gesamtschule gestellt, sollte doch die überwiegende Mehrheit der Schülerinnen und Schüler die neue Hauptschule besuchen. Aber auch die Christlichsozialen waren mit dem Haupt- und Mittelschulgesetz zufrieden, bleib doch die von ihnen so vehement geforderte 
Typenvielfalt im Bereich der Sekundarstufe I bestehen und wurde, was das Realgymnasium betrifft, sogar noch ausgebaut (Grimm, 2008, S. 301). Für Glöckel brachte jedenfalls die Neuregelung den Christlichsozialen einen „Sieg der Namen“, den Sozialdemokraten hingegen einen „Erfolg in der Sache“ (zitiert bei Severinski, 1985, S. 135). Die Frage Vereinheitlichung oder Differenzierung der Schulorganisation im Bereich der Zehn- bis Vierzehnjährigen bleib freilich auch in der Zweiten Republik Österreich (ab 1945) ein bildungspolitischer Streitpunkt zwischen den Sozialdemokraten und der nunmehrigen Österreichischen Volkspartei, der Nachfolgeorganisation der Christlichsozialen Partei (vgl. Engelbrecht, 2014, S. 56-118). Bis heute konnte jedenfalls keine Einigung in dieser so kontrovers diskutierten schul- und gesellschaftspoltischen Thematik erzielt werden. Deshalb ist das österreichische Schulwesen auch gegenwärtig noch durch eine Differenzierung im Bereich der Sekundarstufe I (Neue Mittelschule versus Unterstufe der allgemeinbildenden höheren Schulen) charakterisiert.

\section{Literatur}

ACHS, Oskar und KRASSNIGG, Albert, 1974. Drillschule - Lernschule - Arbeitsschule. Otto Glöckel und die österreichische Schulreform in der Ersten Republik. Wien: Jugend und Volk. ISBN 3-7141-5346-2.

ADAM, Erik, 2007. Die österreichische Reformpädagogik - eine verschüttete Tradition. In: KNAPP, Gerald und LAUERMANN, Karin, ed. Schule und Soziale Arbeit. Zur Reform der öffentlichen Erziehung und Bildung in Österreich. Klagenfurt, Ljubljana und Wien: Hermagoras, S. 26-44. ISBN 978-3-7086-0338-4.

BALLAUFF, Theodor und SCHALLER, Klaus, 1973. Pädagogik. Eine Geschichte der Bildung und Erziehung. Bd. III: 19./20. Jahrhundert. Freiburg i. Br. und München: Alber. ISBN 3-495-47222-3.

BREZINKA, Wolfgang, 2000. Pädagogik in Österreich. die Geschichte des Faches an den Universitäten vom 18. bis zum Ende des 20. Jahrhunderts. Bd. 1: Einleitung, Pädagogik an der Universität Wien. Wien: Verlag der Österreichischen Akademie der Wissenschaften. ISBN 3-70012908-4.

BREZINKA, Wolfgang, 2003. Pädagogik in Österreich. die Geschichte des Faches an den Universitäten vom 18. bis zum Ende des 20. Jahrhunderts. Bd. 2: Pädagogik an den Universitäten Prag, Graz und Innsbruck. Wien: Verlag der Österreichischen Akademie der Wissenschaften. ISBN 3-7001-3218-2.

DACHS, Herbert, 1982. Schule und Politik. Die politische Erziehung an den österreichischen Schulen 1918 bis 1938. Wien: Jugend und Volk. ISBN 3-224-16506-5.

DAHMS, Hans-Joachim und STADLER, Friedrich, 2015. Die Philosophie an der Universität Wien von 1848 bis zur Gegenwart. In: KNIEFACZ, Katharina et al., ed. Universität Forschung - Lehre. Themen und Perspektiven im langen 20. Jahrhundert. Göttingen: V \& R unipress, S. 77-131. ISBN 978-3-8471-0290-8. 
ENGELBRECHT, Helmut, 1988. Geschichte des österreichischen Bildungswesens. Erziehung und Unterricht auf dem Boden Österreichs. Bd. 5: Von 1918 bis zur Gegenwart. Wien: Österreichischer Bundesverlag. ISBN 3-215-06054-X.

ENGELBRECHT, Helmut, 2014. Unendlicher Streit durch Jahrhunderte. Vereinheitlichung oder Differenzierung in der Organisation österreichischer Schulen. Wien: New Academic Press. ISBN 978-3-7003-1909-2.

GLÖCKEL, Otto, 1916. Einheitsschule. In: GLÖCKEL, Otto, 1985. Ausgewählte Schriften und Reden, ed. und ACHS, Oskar. Wien: Jugend und Volk, S. 91-95. ISBN 3-224-1-5300-8.

GLÖCKEL, Otto, 1917, Das Tor der Zukunft. In: GLÖCKEL, Otto, 1985. Ausgewählte Schriften und Reden, ed. und ACHS, Oskar. Wien: Jugend und Volk, S. 99-121. ISBN 3-2241-5300-8.

GLÖCKEL, Otto, 1919, Die nächsten Pläne der Schulverwaltung. Ausführungen des Unterstaatssekretärs für Unterricht am 22. April 1919. In: GLÖCKEL, Otto, 1985. Ausgewählte Schriften und Reden, ed. und ACHS, Oskar. Wien: Jugend und Volk, S. 141147. ISBN 3-224-1-5300-8.

GÖNNER, Rudolf, 1967. Die österreichische Lehrerbildung von der Normalschule bis zur Pädagogischen Akademie. Wien: Österreichischer Bundesverlag.

GRIMM, Gerald, 2007. Demokratisierung und Liberalisierung. Zur Geschichte der Schulreform Otto Glöckels in Österreich. In: KNAPP, Gerald und LAUERMANN, Karin, ed. Schule und Soziale Arbeit. Zur Reform der öffentlichen Erziehung und Bildung in Österreich. Klagenfurt, Ljubljana und Wien: Hermagoras, S. 45-69. ISBN 978-3-7086-0338-4.

GRIMM, Gerald, 2008. Schulpolitik und Schulmodelle: Anspruch, Anforderungen und Realität. In: KARNER, Stefan und MIKOLETZKY, Lorenz, ed. Österreich. 90 Jahre Republik. Beitragsband der Ausstellung im Parlament. Innsbruck, Wien und Bozen: StudienVerlag, S. 295-305. ISBN 978-3-7065-4664-5.

GRIMM, Gerald, 2018. Das Modell einer „Zentralmatura“: Ein Novum im österreichischen Bildungswesen?. In: GÖTTLICHER, Wilfried, LINK, Jörg-W. und MATTHES, Eva, ed. Bildungsreform als Thema der Bildungsgeschichte. Bad Heilbrunn: Klinkhardt, S. 151-166. ISBN 978-3-7815-2214-5.

HACKL, Bernd, 1990. Die Arbeitsschule. Geschichte und Aktualität eines Reformmodells. Wien: Verlag für Gesellschaftskritik. ISBN 3-85115-117-8.

HÖBELT, Lothar, 2018. Die Erste Republik Österreich (1918-1938). Das Provisorium. Wien, Köln und Weimar: Böhlau. ISBN 978-3-205-20539-5.

HÖFLER, Alois, 1906. Gymnasium. In: LOOS, Joseph, ed. Enzyklopädisches Handbuch der Erziehungskunde. Wien und Leipzig: Pichler, S. 628-645.

HÖFLER, Alois, 1908. Drei Vorträge zur Mittelschulreform. Wien und Leipzig: Braumüller. HÖFLER, Alois, 1916. „Die deutsche höhere Schule nach dem Weltkriege“. Und die österreichische? Zeitschrift für die österreichischen Gymnasien. 67, S. 712-741.

HÖFLER, Alois, 1918. Das Ganze der Schulreform in Österreich. Stichproben und Ausblicke. Leipzig, Prag und Wien: Haase. 
HÖFLER, Alois, 1921. Die Philosophie des Alois Höfler. In: SCHMIDT, Raymund, ed. Die deutsche Philosophie der Gegenwart in Selbstdarstellungen. 2. Bd., Leipzig: Meiner, S. 117160.

HÖFLER, Alois und MEISTER, Richard, 1921. Audiatur et altera pars. In: Volkserziehung. Nachrichten des Österreichischen Unterrichtsamtes. Pädagogischer Teil, S. 283-293, 311323, 479-493.

HÖRBURGER, Franz, 1967. Geschichte der Erziehung und des Unterrichts. Wien und München: Österreichischer Bundesverlag.

KRIECHBAUMER, Robert, 2017. Die Christlichsoziale Partei 1918 bis 1933/34. In: KARNER, Stefan, ed. Die umkämpfte Republik. Österreich 1918-1938. Innsbruck, Wien und Bozen: StudienVerlag, S. 137-145. ISBN 978-3-7065-5637-8.

LECHNER, Elmar, 1995. Die hohe Zeit der bildungspolitischen Ideologien - Korporativer Patriarchalismus und etatistischer Sozialismus im Meinungs- und Machtkampf. In: TÀLOS, Emmerich et al., ed. Handbuch des politischen Systems Österreichs. Erste Republik 19181933. Wien: Manz, S. 504-527. ISBN 978-3214059637.

MEISTER, Richard, 1919/1946. Unterrichtsfächer als Dispositionssysteme (1919). In: MEISTER, Richard, 1946. Beiträge zur Theorie der Erziehung. Wien: Verlag Ringbuchhandlung A. Sexl, S. 76-91.

MEISTER, Richard, 1921/1946. Lehrfächer und Wissenschaften, Ein Beitrag zu den Problemen der Überbürdung und Konzentration (1921). In: MEISTER, Richard, 1946. Beiträge zur Theorie der Erziehung. Wien: Verlag Ringbuchhandlung A. Sexl, S. 69-75.

MEISTER, Richard. 1924/1946. Die Erörterung über den Wissenschaftscharakter der Pädagogik in der neueren Forschung (1924). In: MEISTER, Richard, 1946. Beiträge zur Theorie der Erziehung. Wien: Verlag Ringbuchhandlung A. Sexl, S. 9-19.

OLECHOWSKI, Richard, 2014. Die „Kulturpädagogik“ - ein Weg in die Erstarrung durch Konservatismus? Zum 50. Gedenktag des Todestags von Richard Meister. Erziehung und Unterricht. Österreichische pädagogische Zeitschrift, 164, S. 521-529. ISSN 0014-0325.

REBLE, Albert, 1989. Geschichte der Pädagogik. 15., neu bearbeitete, Auflage. Stuttgart: Klett-Cotta. ISBN 3-608-93011-6.

SEEL, Norbert M. und HANKE, Ulrike, 2015. Historische Pädagogik: die Geschichte der Erziehung und der Erziehungswissenschaft. In: SEEL, Norbert M. und HANKE, Ulrike. Erziehungswissenschaft. Lehrbuch für Bachelor-, Master- und Lehramtsstudierende. Berlin und Heidelberg: Springer VS, S. 157-305. ISBN 978-3-642-55205-2.

SCHEIPL, Josef, 2017. Österreichische Schulpolitik 1918 bis 1938 - Parteipolitische und weltanschauliche Hintergründe. In: KARNER, Stefan, Hrsg. Die umkämpfte Republik. Österreich 1918-1938. Innsbruck, Wien und Bozen: StudienVerlag, S. 321-326. ISBN 9783-7065-5637-8.

SEVERINSKI, Nikolaus, 1985. Die Gesamtschulidee. Historischer Aufriß mit besonderer Berücksichtigung der ,,Glöckelschen Schulreform “ in Österreich. Wien und Salzburg: GeyerEdition. Verbund-ID-Nr. AC 00576145. 
SKIERA, Ehrenhard, 2018. Erziehung und Kontrolle. Über das totalitäre Erbe in der Pädagogik im ,Jahrhundert des Kindes ' Bad Heilbrunn: Klinkhardt. ISBN 978-3-7815-22541.

WALLRAF, Udo, 1986. Kultur und Persönlichkeit. Richard Meister als Erziehungstheoretiker und Reformer des österreichischen Bildungswesens. Krefeld: Pädagogik \& Hochschul-Verlag. Verbund-ID-Nr. AC00050149. 